\title{
Outcome of Multidrug Resistant Tuberculosis at Tertiary Care Hospital, Rahim Yar Khan
}

\author{
Hafiz Muhammad Rizwan, ${ }^{1}$ Hafiza Shafia Naz, ${ }^{1}$ Imran Bashir, ${ }^{1}$ Arsalan Ahmad Khan Durrani, ${ }^{1}$ Shazia Mehmood, \\ Masood Ul Haq ${ }^{1}$
}

\begin{abstract}
Background: In patients of Multi drug resistant (MDR) TB, proper counseling of MDR-TB patients, regarding adherence to therapy, monitoring side effects and controlling the comorbidities, lead to better out come.

Objective: To determine the outcome of MDR-TB at a tertiary care hospital in Rahim Yar Khan.

Methodology: This retrospective cohort study was carried out at Department of Pulmonology, Sheikh Zayed Hospital, Rahim Yar Khan. A total of 231 patients of pulmonary MDR, were included from May 2014 to December 2017, patients having age between 10 to 80 years of either sex. Extra pulmonary DR-TB, Poly drug resistant TB, $\mathrm{XDR}$ and patients on shorter regimen of DR -TB were excluded from the study. Treatment outcomes like cured, treatment complete, died, treatment failure and lost to follow up were defined according to WHO definitions. Data was then analyzed with SPSS version 22.0

Results: Out of 231 patients, $123(57.1 \%)$ were male, with mean age of $36.13+16$ years. Regarding treatment outcome, 115 (49.8\%) patients cured, 1 (0.4\%) completed, 67 (29.0\%) patients died, $5(2.2 \%)$ patients showed treatment failure, $6(2.6 \%)$ patients lost to follow up, 27 (11.7\%) patents are still under treatment and treatment of 10 (4.3\%) patients not evaluated. Over all successful outcome (treatment completed and cured) was 50.2\% while unsuccessful outcome (died, treatment failure, lost to follow up, painters still under treatment and treatment not evaluated) was $49.8 \%$.
\end{abstract}

Conclusion: Successful outcome was noted in half of the pulmonary MDR-TB patients which is close to WHO target success rate. Younger age groups showed significantly better outcome.

Key words: MDR, Tuberculosis, Outcome

\section{Introduction}

Multidrug - resistant tuberculosis is a form of tuberculosis, which is caused by a strain of mycobacterium tuberculosis, that is resistant to at least, two of the most effective, first line Anti-TB drugs i.e. Isoniazid \& Rifampicin. ${ }^{1}$ Drug resistant TB continues to be a major public health crisis. Worldwide in 2017, 55,8000 people developed tuberculosis, that was resistant to Rifampicin (RRTB), \& of these patients, $82 \%$ were diagnosed as multidrug-resistant tuberculosis. Globally, 3.5\% of new TB cases \& 18\% of previously treated cases had MDR-TB. ${ }^{2}$ Pakistan is currently ranked $4^{\text {th }}$ among the highest MDR-TB burden countries. ${ }^{3}$ As MDR-TB patients are managed with more expensive and toxic second line Anti-TB drugs and may require hospital admission for management of side effects as well as other complication associated with these drugs, a significant portion of health budget is being utilized on these patients. Due to complexity of this problems and difficult management of drugresistant $\mathrm{TB}$, national $\mathrm{TB}$ control program decided to control this major health issue through a systematic and programmatic management of drug-resistant $\mathrm{TB}$ (PMDT) all over the country. ${ }^{4}$ Estimation of MDR$\mathrm{TB}$ in Pakistan is variable. A review throughout the country showed that $24.3 \%$ of the previously treated cases were MDR-TB. ${ }^{5}$ MDR-TB is a man-made problem. ${ }^{6}$ Improper and mismanagement of MDRTB may lead to the development of extensively drugresistant TB XDR-TB. ${ }^{7}$ Outcome of MDR-TB management depends upon adequate information on epidemiological factors, patient education, duration of treatment, response of treatment during the course, side effect profile of medicines, reasons for default from previous treatment and regular followup visits. ${ }^{8}$

Department of Pulmonology, Sheikh Zayed Medical College / Hospital, Rahim Yar Khan is serving the patients of chest diseases in a very remote district of Punjab on indoor and outdoor basis. Being situated in

1.Department of Pulmonology, Sheikh Zayed Medical College/Hospital, Rahim Yar Khan, University of Health Sciences Lahore, Pakistan.

Correspondence: Dr. Hafiz Muhammad Rizwan, Assistant Professor, Department of Pulmonology, Sheikh Zayed Medical College/Hospital, Rahim Yar Khan, Pakistan.

Email: hafiz_rizwan_u@yahoo.com Phone:+92-333-6336700 Received: 21-05-2019

Accepted: 14-11-2019 Published: 25-12-2019 
the farthest district of province Punjab, this hospital is also providing health services to the patients of other provinces like Sindh and Baluchistan for drug-susceptible as well as drugresistant TB. PMDT site started its work in Rahim Yar Khan in 2014. Before that, DR-TB patients were being managed privately on individual basis and there was no proper record keeping system for these patients. With start of PMDT site things were streamlined in terms of patients registration as well as their record keeping in all aspects of DRTB management. Up till now no such study was done in this large populated area on this important aspect of DR-TB, that's why we conducted this study in our tertiary care hospital in collaboration with PMDT site, with objective to determine the outcome of MDR-TB at a tertiary care hospital in Rahim Yar Khan.

\section{Methodology}

In this retrospective cohort, a total of 231 patients of Pulmonary MDR TB, confirmed on sputum GeneXpert from May 2014 to Dec 2017, between the ages of 10 to 80 years of either sex were included. This study was conducted with the approval obtained from Institutional Review Board of Sheikh Zayed Medical College / Hospital, Rahim Yar Khan. Patients with ExtraPulmonary TB, Poly Drug Resistant TB, Extensively Drug Resistant TB (XDR TB) and those who were registered for short term regimen of MDR TB after December 2017, were excluded from this study. Pretreatment investigations included complete Blood count, ESR, Renal and Liver function test, Serum Electrolytes, Blood sugar, Serum Uric Acid, Viral Markers (HBsAg, Anti-HCV), HIV and TSH. Audiometery and visual tests were performed every month in the intensive phase. Chest X-ray was done at the start of treatment and then every third month. Duration of treatment was 18-24 months and all the patients were followed up every month for upto 24 months. Sociodemographic data was recorded on predesigned performa. All the data was then analyzed with SPSS version 22. Mean \pm SD were taken for quantitative variable like age. Qualitative variable like gender and different outcomes were presented as frequencies and percentages. Treatments outcomes were labelled as cured, treatment completed, died, treatment failure and lost to follow-up, according to WHO guidelines. ${ }^{9}$ Treatment outcomes were further grouped as:

1. Successful (Treatment Completed and Cured)

2. Unsuccessful (Died, Treatment Failure, Lost to follow up, Still under Treatment and Treatment not evaluated).

\section{Results}

In this study, out of 231 patients, 132 (57.1\%) were males and $99(42.9 \%)$ were females with mean age of $36.13 \pm 16$ years. Majority of patients 187 (81\%) were from District Rahim Yar Khan and 44 patients $(19 \%)$ were residents of other districts. One Hundred and Eleven $(48.1 \%)$ patients had resistance to INH and Rifampicin only while $120(51.9 \%)$ patients had resistance to one or more drugs in addition to $\mathrm{INH}$ and Rifampicin. Treatment outcome is shown in table I.

Figure I: Overall Treatment Outcome in MDR TB Patients $(n=231)$

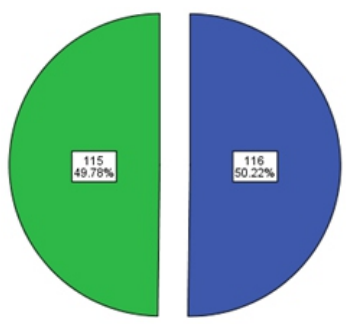

Treatment outcome was further classified as successful $116(50.2 \%)$ or unsuccessful 115 (49.8\%) according to our study protocol. (Figure I) Analysis of successful or unsuccessful treatment outcome with reference to gender, age distribution, patients of district Rahim Yar Khan to other districts and drug resistance to two drugs or more than two drugs is shown in table II.

Table I: Treatment Outcome of MDR TB Patients $(\mathrm{n}=\mathbf{2 3 1})$

\begin{tabular}{|l|c|c|}
\hline \multicolumn{1}{|c|}{ Outcome } & Frequency & Percentage \\
\hline Complete & 1 & $0.4 \%$ \\
\hline Cured & 115 & $49.8 \%$ \\
\hline Died & 67 & $29.0 \%$ \\
\hline Failed & 5 & $2.2 \%$ \\
\hline Lost to follow up & 6 & $2.6 \%$ \\
\hline Still under Treatment & 27 & $11.7 \%$ \\
\hline Treatment not evaluated & 10 & $4.3 \%$ \\
\hline Total & 231 & 100.0 \\
\hline
\end{tabular}


Table I : Sociodemographic Characteristics and Outcome of MDR TB patients $(n=231)$

\begin{tabular}{|c|c|c|c|}
\hline & Successful & Unsuccessful & $\begin{array}{c}\mathbf{P} \\
\text { Value }\end{array}$ \\
\hline $\begin{array}{l}\text { Overall } \\
\text { outcome }\end{array}$ & 116 & 115 & \\
\hline \multicolumn{4}{|l|}{ Sex } \\
\hline Male & 70 & 62 & \multirow{2}{*}{0.323} \\
\hline Female & 46 & 53 & \\
\hline \multicolumn{4}{|c|}{ Age group (years) } \\
\hline 10 to 30 & 59 & 45 & \multirow{4}{*}{0.027} \\
\hline 31 to 50 & 44 & 42 & \\
\hline 51 to 70 & 13 & 24 & \\
\hline$>70$ years & 00 & 04 & \\
\hline \multicolumn{4}{|l|}{ Residents } \\
\hline $\begin{array}{l}\text { District Rahim } \\
\text { Yar Khan }\end{array}$ & 92 & 95 & \multirow{2}{*}{0.523} \\
\hline Other District & 24 & 20 & \\
\hline \multicolumn{4}{|c|}{ Drug resistance to } \\
\hline $\begin{array}{l}\text { INH \& } \\
\text { Rifampicin } \\
\text { Only }\end{array}$ & 56 & 55 & \multirow{2}{*}{0.945} \\
\hline $\begin{array}{l}\text { INH + } \\
\text { Rifampicin + } \\
\text { Other Drugs }\end{array}$ & 60 & 60 & \\
\hline
\end{tabular}

\section{Discussion}

In the present study, $116(50.2 \%)$ patients showed successful outcome, which is close to WHO target success rate of MDR treatment i.e. 55\%. Different studies from various countries (India, Tamil nadu, Russia and USA) have reported higher successful rates varying from 50 $70 \% .{ }^{11,12,13,14}$ In Pakistan, treatment outcome is also variable in different parts of the country. Khan et al. in 2015, concluded that treatment success rate among the MDR TB patients, studied between $2008-2011$ in Khyber Pakhtunkhwa was $63 \%{ }^{15}$ Another study from Khyber Pakhtunkhwa reported the success rate of $74.3 \%{ }^{16}$ Similarly a study from Karachi reported 39.2\% success rate of the disease. ${ }^{17}$

In our study, we also analyzed treatment outcomes with reference to gender, age distribution, patients of District Rahim Yar Khan versus from patients of other districts and number of drug resistance (2 or more than 2 drug resistance). If we talk about gender, successful treatment outcome among the males was higher $(60.3 \%)$ as compared to females $(39.7 \%)$ which may be explained by male dominance in our society and there is less attention towards the female population for seeking medical advice in case of their health related problems. When different age groups were analyzed with reference to treatment outcome, success rate was higher among the patients of aged $10-30$ years that lowered down in $30-50$ year of age group and then so on. P-value for different age groups was statistically significant $(\mathrm{P}$-value $=$ 0.027). This may be explained by absence of comorbidities in this age group $(10-30$ years $)$ and better compliance to treatment. As the age advances, people usually suffer from various co-morbidities affecting the treatment outcome. So early diagnosis in young age group results in better outcome.

No significant difference was found in treatment outcome among the patients of district Rahim Yar Khan and patients coming from other districts. Similarly, no significant difference was observed when treatment outcome was analyzed with reference to resistance to 02 drugs (INH and Rif) and $>2$ drugs containing INH and Rifampicin.

In our study, the case fatality rate was $29 \%$, which is comparable to other studies. ${ }^{18,19,20}$ Possible factors responsible for high death rate in our study may be strict inclusion criteria (only Pulmonary MDR cases taken), late diagnosis of the disease and presence of co-morbidities (although we did not study the comorbidities). Treatment failure and default rates were $2.2 \%$ and $2.6 \%$ respectively, in our study which are very reasonable as compared to a study done in India in 2015 in which Sangita V. et al showed treatment failure $06 \%$, lost to follow up $21 \%$ and death rate $30 \%{ }^{21}$ The significant low treatment failure and default rate in our study are due to certain reasons. Firstly, we have treatment coordinator at our PMDT site for communication with the patients. Secondly, we have a psychologist who counsels the patients of MDR TB as well as their attendants regarding disease explanation and importance of adherence of therapy. Thirdly, in some difficult 
cases, even our MDR physician, himself visits the patient's homes to facilitate their problems regarding treatment and follow up.

We could not evaluate treatment outcome in 10 patients as some patients were transferred out to other PMDT site due to their family shifting and some patients did not attend the call deliberately or changed their contact number. Our study has certain limitation. We did not study the extent of disease, duration of illness prior to the diagnosis and co-morbidities associated with MDR TB. Further studies should be planned to investigate above mentioned points. Furthermore, short course regimens have been started at our PMDT site, so two regimes (Longer vs Shorter) can be compared.

\section{Conclusion}

The successful outcome was found among half of the Pulmonary MDR TB, which is close to WHO target. Younger patients showed significantly better outcomes. Still half of MDR patients have poor (unsuccessful) outcome, which needs to be improved by addressing the responsible factors.

Authors Contribution: HMR:Conception, design of work, interpretation and drafting. HSN: Acquisition and revising.IB:Acquisition and analysis and revising. AAKD:Interpretation and revising. SM:Acquisition, Interpretation and revising. MH: Conception, interpretation and drafting. All the authors gave the final approval for publishing and agreed to be accountable for all aspect of work.

\section{Conflict of Interest: None \\ Sources of Funding: Self}

\section{References}

1. World Health Organization. "Diagnosis and notification of multidrug-resistant TB" (PDF). WHO MDR TB Factsheet (2016 Update). Available from: http://www.who.int/ tb/challenges $/ \mathrm{mdr} / \mathrm{mdr}$ tb factsheet.pdf

2. World Health Organization. Global Tuberculosis Report 2018.

3. World Health Organization. WHO 2016 global report on country profiles for the 30 - high burden countries for tuberculosis. Available from : ht t p :// w w w. who.int / t b / publications/global_report/gtbr2016_annex2.

4. National guidelines for the management of Drug Resistant Tuberculosis: National TB Control Program, Islamabad; 2009.

5. Javaid A, Hasan R, Zafar A, ChaudryMA, Qayyum S, Qadeer E, Shaheen Z, Agha N, Rizvi $\mathrm{N}$, et al. Pattern of first-and second-line drug resistance among pulmonary tuberculosis retreatment cases in Pakistan. The International Journal of Tuberculosis and Lung Disease. 2017;21(3): 303-8.

6. Kant S, Maurya Ak, Kushwaha RA, Nag VL, Prasad P. Multi-Drug resistant tuberculosis: an iatrogenic problem. Bio Sci. Trends 2010;4(2):48-55.

7. Ullah I, Javaid A, Tahir Z, Ullah O, Shah AA, Hasan F, Ayub N. Pattern of drug resistance and risk factors associated with development of drug resistant Mycobacterium tuberculosis in Pakistan. Plos one. 2016 Jan 25;11(1):e0147529.

8. Khan MA, Mehreen S, Basit A, Khan RA, Javaid A. Predictors of poor outcomes among patients treated for multidrug-resistant tuberculosis at Tertiary Care Hospital in Pakistan. AmericanEurasian J Toxicol Sci. 2015;7(3):162-72.

9. Companion handbook to the WHO guidelines for the programmatic management of drugresistant tuberculosis. Geneva, Switzerland: World Health Organization; 2014.

10. World Health Organization. Global Tuberculosis Report 2017.

11. Datta BS, Hassan G, Kadri SM, Qureshi W, Kamili MA, Singh H, et al. Multidrugresistant and extensively drug resistant tuberculosis in Kashmir, India. J Infect Dev Ctries 2010;4(1):19-23.

12. Joseph P, Desai VB, Mohan NS, Fredrick JS, Ramachandran R, Raman B, et al. Outcome of standardized treatment for patients with MDR-TB from Tamil Nadu, India. Indian J Med Res 2011;133:529-34.

13. Cavanaugh JS, Kazennyy BY, Nguyen ML, Kiryanova EV, Vitek E, Khorosheva TM, et al. Outcomes and follow-up of patients treated for multidrug-resistant tuberculosis in Orel, Russia, 2002-2005. Int J Tuberc Lung Dis 2012;16(8):1069-74. 
14. Kurbatova EV, Taylor A, Gammino VM, Bayona J, Becerra M, Danilovitz M, et al. Predictors of poor outcomes among patients treated for multidrug-resistant tuberculosis at DOTS-plus projects. Tuberculosis (Edinb) 2012;92(5):397-403.

15. Khan MA, Basit A, Javaid A, Adnan MA, Ahmad F, Khan N, Khattak A, Sajjad H, Khattak MA. Outcome of community based therapy of Multi-Drug Resistant tuberculosis patients treated in a tertiary care hospital of Khyber Pakhtunkhwa. Pakistan Journal of Chest Medicine. 2015;20(3).

16. Khan MA, Mehreen S, Basit A, Khan RA, Jan F, Ullah I, Ihtesham M, et al. Characteristics and treatment outcomes of patients with multi-drug resistant tuberculosis at a tertiary care hospital in Peshawar, Pakistan. Saudimedical journal.2015Dec;36(12):1463.

17. Rao NA, Mahfooz Z, Irfan M. Treatment outcome of multi-drug resistant tuberculosis in a tertiary care hospital in Karachi. Journal of Pakistan Medical Association. 2009;59(10):694.
18. Leimane V, Dravniece G, Riekstina V, Sture I, Kammerer S, Chen MP, et al. Treatment outcome of multidrug/extensively drugresistant tuberculosis in Latvia, 2000-2004. Eur Respir J 2010;36(3):584-93.

19. Johnston JC, Shahidi NC, Sadatsafavi M, Fitzgerald JM. Treatment outcomes of multidrug-resistant tuberculosis: a systematic review and meta-analysis. PLoS ONE 2009;4(9):e6914.

20. Shin SS, Pasechnikov AD, Gelmanova IY, Peremitin GG, Strelis AK, Mishustin S, et al. Adverse reactions among patients being treated for MDR-TB in Tomsk, Russia. Int J Tuberc Lung Dis 2007;11(12):1314-20.

21. Sangita V, Patel, Kapil B, Nimavat, Patel B, Alpesh, et al. Treatment outcome among cases of multidrug-resistant tuberculosis (MDR TB) in Western India: A prospective study. Journal of Infection and Public health. 2016;9:478-84.

Article Citation: Rizwan HM, Naz HS, Bashir I, Durrani AAK, Mehmood S, Haq M. Outcome of Multidrug Resistant Tuberculosis at a Tertiary Care Hospital, Rahim Yar Khan. JSZMC 2019;10(4):08-12 